can incorps:ate as much as 12 minutes of film, 30000 slides, and an dudio channel; the disk is controlled by a microcomputer. Students can choose whether to progress through the programme as it appears on the screen, to choose specific topics, or to consult one element, such as slides or film. They can respond to the questions asked on the commentary, a feature also found in the software packages for computer assisted learning. These are proving popular with students, who enjoy working through them at their own speed, and who are left in no doubt by the programme when they have pursued a course that would be fatal to an actual patient.

GILLIAN M PENTELOW

Librarian,

King's College School of Medicine and Dentistry,

London SE5 9PJ

1 Smith J. A new tool in the fight against AIDS? Br Med $\mathcal{F} 1988 ; 297: 9$

\title{
Hepatitis B: prevention in primary care
}

\section{More community based policies needed}

Hepatitis B is rarely seen by most general practitioners in Britain for, though there may be 300 million carriers of active infection in the world, ${ }^{1}$ it is not endemic in the West. Nevertheless, two fifths of children with persistent infection who survive to adulthood die as a result of chronic liver disease and carcinoma, ${ }^{1}$ and both active and passive immunisation against the disease are now effective; thus a policy for preventing infection is essential. We believe that this requires not only good national protocols with audit ${ }^{2}$ but also coherent community based policies for education, consent, testing, immunisation, and follow up. ${ }^{3}$

Two different populations are at high risk of hepatitis B. The first consists of people who, by the nature of their activities are at risk from blood borne or sexual transmission. ${ }^{4}$ These include intravenous drug abusers, some homosexual men, and some health workers as well as carers and their clients in residential institutions for the mentally handicapped, where close contact with body fluids may occur. The general practitioner is in a good position to advise these people about risk reduction and immunise when necessary. The second population consists of people from several immigrant groups from parts of the world with high carrier rates - up to $20 \%$ in South East Asia - that provide pockets of endemicity, with sexual, vertical, and household transmission of infection. Contamination of the blood of the newborn is very likely to be followed by persistent carriage of the virus, and there are ethnic differences in the likelihood of vertical transmission, which is particularly high in Chinese families. ${ }^{5}$ Here an approach through the family and primary care is essential.

This group includes the Vietnamese and Chinese boat people, two fifths of whom in the United Kingdom are now carriers of hepatitis B ( $\mathrm{M}$ J Masters, unpublished findings). Some 20000 such refugees are scattered widely throughout Britain; most have young families, and most are still mobile. Many have experienced, although not necessarily understood, the antenatal screening programme leading to immunisation of neonates at risk. ${ }^{2}$ This aims at preventing vertical transmission and so reducing the carrier rate, but it is a hospital based programme for combating a disease that occurs within a community. Thus good communication with primary care services is vital. There should be clear protocols integrating the neonatal programme into the general health care immunisation schedules, with boosters given in child health clinics, so that the timing fits in with that for the other routine immunisations. A recent study has shown excellent rates of seroconversion when hepatitis $B$ vaccine is given at the same time as the triple vaccines. ${ }^{6}$ There should be formal consent procedures, records held by the patients, and information in as many languages as necessary. For effective coverage there must be a joint approach among general practitioners, health visitors, and midwives and there must be linkworkers who can explain and interpret to people with different languages and cultures. Health workers and, more importantly, those at risk need to understand about the disease and its transmission, carriage, and prevention. A few people will always refuse to be immunised, but we can reduce general fear and ignorance about hepatitis $B$ and the vaccine.

Hepatitis B is a disease of intimacy and so of the family unit, ${ }^{7}$ and hence the community team has a unique role in tackling it. The need for systematic household screening of high risk groups remains problematic as no treatment has proved efficacy for carriers. Nevertheless, screening the families and the contacts of carriers would benefit people susceptible to the disease, who could be offered immunisation. If family trees are submitted with the samples the virologist may be able to give more detailed information on the levels of risk and whom to immunise.

Informed consent to such screening is needed. Known carriers and their families need counselling in relation to handling body fluids, blood spillage, medical and dental treatment, and vertical and sexual transmission. Active immunisation of sexual partners is recommended, as well as counselling to encourage use of barrier methods of contraception.

NIGEL MASTERS

Lecturer,

Department of General Practice,

United Medical and Dental Schools,

Guy's Campus,

London SE1 9RT

General Practitioner,

Gill Street Health Centre,

London E14 8HQ

ANNA LIVINGSTONE

Health Visitor,

Gill Street Health Centre,

London E14 8HQ

VIVIENNE CENCORA

\footnotetext{
1 World Health Organisation. Progress in the control of viral hepatitis: memorandum from WHO meeting. Bull WHO 1988;66:443-55

2 Polakoff S, Vandervelde EM. Immunisation of neonates at high risk of hepatitis in England and Wales; national surveillance. Br Med $\mathcal{J}$ 1988;297:249-53.

3 Cencora V, Challoner F, Gooch S, Livingstone A, Widgery D. Immunisation of neonates at risk of hepatitis B. Br Med F 1988;297:481.

4 Clee WB, Hunter PR. Hepatitis B in general practice: epidemiology, clinical and serological features, and control. BrMed f 1987;295:530-2.

5 Polakoff S. National surveillance of immunisation of infants at risk of acquiring hepatitis B virus infection. Revised protocol. London: Hepatitis Epidemiology Unit, Public Health Laboratory Service, 1988

6 Piazza $M$, Da Villa G, Picciotto $\mathrm{L}$, et al. Mass vaccination against hepatitis B in infants in Italy. Lancet 1988;ii: 1132 .

7 Nordenfelt E, Dahlquist E. HBsAg positive adopted children as a cause of intrafamilial spread of hepatitis B. Scand F Infect Dis 1978;10:161-3.
} 